\title{
Colpevolezza ed esagerazione nell'interpretazione del terrorismo brigatista da parte della stampa italiana
}

\author{
Matteo $\mathrm{RE}^{1}$ \\ Universidad Rey Juan Carlos
}

Recibido: 03/10/2010

Aceptado: 11/03/2011

\section{RIASSUNTO}

In questo articolo si analizza il modo in cui la stampa nazionale italiana trattò il tema delle Brigate Rosse nel periodo che va dagli anni settanta all'inizio degli anni ottanta. In questo periodo temporale è possibile osservare come la maggior parte dei giornalisti italiani commise il grave errore di sottovalutare la potenza criminale del gruppo terrorista e si ostinò a collocare la banda armata ideologicamente tra i gruppi eversivi neofascisti, quando invece gli stessi brigatisti dichiaravano reiteratamente il loro marxismo-leninismo.

Nella seconda parte dell'articolo, si parla di come vennero analizzati, da parte dei principali periodici italiani, il sequestro e l'assassinio dell'onorevole Aldo Moro e di come le Brigate Rosse, durante il rapimento del magistrato D'Urso, cercarono di utilizzare i mezzi di comunicazione per i loro fini criminali.

Parole chiave: Terrorismo, giornalismo, Brigate Rosse.

Culpability and exageration of the interpretation of the Red Brigades terrorism by the Italian mass media.

\begin{abstract}
In this article the author analyses the way in which the Italian mass media dealt with the issue of the Red Brigades from the beginning of the decade of the seventies and the first half of the eighties. It is possible to perceive how the majority of the Italian journalists made the enormous mistake of undermining the criminal power of the terrorist group during this
\end{abstract}

${ }^{1}$ Universidad Rey Juan Carlos. Departamento de Lenguas Modernas, despacho 102, Edificio Departamental, Camino del Molino s/n, 28943, Fuenlabrada-Madrid. matteo.re@urjc.es. 
period and how they placed the armed band ideologically among the neo-fascist groups, although the own members of the brigades declared their loyalty to the Marxism-Leninism many times.

The second part of the article deals with the kidnapping and murder of the president of the political party «Democrazia Cristiana», Aldo Moro, and how, during the captivity of the magistrate D'Urso, the Red Brigades tried to use the Italian media for their own criminal purposes.

Key words: Terrorism, media, journalism, Red Brigades.

\section{INTRODUZIONE}

Sono passati quasi tre decenni da quei fatidici anni settanta che in Italia, molte volte a torto, si ricordano ancora oggi quasi unicamente come gli «anni di piombo», come un oscuro periodo di barbarie in cui tutto era permesso e del quale noi italiani continuiamo a portare sulle spalle il fardello. In realtà, quei momenti furono caratterizzati anche da un notevole sviluppo riformista che interessò le più ampie sfere sociali del paese: dopo un lungo iter, venne approvata la legge sul divorzio, grazie a un referendum celebrato nel maggio del 1974; più tardi fu la volta della riforma della legge sulla famiglia, con la quale la donna e i figli acquistavano più diritti all'interno del nucleo familiare; quindi ci fu l'abbassamento della maggiore età a 18 anni e, infine, si arrivò, nel 1978, alla legge sull'interruzione volontaria della gravidanza. È vero comunque che quella epoca fu caratterizzata da un aumento spropositato della violenza che, per attenuarne forse la gravità, si faceva ricondurre alla politica, come se l'omicidio dettato da ragioni ideologiche fosse meno meschino di quello mosso da altri motivi.

Si cominciò con un periodo di aspra protesta giovanile che si ricorda con il numero dell'anno in cui arrivò al suo culmine, il sessantotto. Poi si proseguì con il malcontento nelle fabbriche e con le lotte operaie per i rinnovi dei contratti collettivi di lavoro. Gli anni sessanta si conclusero in maniera drammatica con uno degli attentati più atroci mai commessi sul territorio italiano: il 12 dicembre 1969 nella hall della Banca Nazionale dell'Agricoltura di Milano scoppiò una bomba che provocò la morte di 17 persone. Le indagini si concentrarono sull'anarchia milanese. Il ferroviere anarchico Giuseppe Pinelli, accusato di essere 1'autore della strage, durante l'interrogatorio in questura precipitò dalla finestra della stanza in cui si trovava. Dopo molte illazioni, ricerche, inchieste si dimostrò che il malcapitato era caduto in maniera accidentale e non spinto dagli uomini di Luigi Calabresi, il commissario che si occupava dell'inchiesta. Questa versione dei fatti non convinse quasi nessuno e provocò accese polemiche, tanto più quando si scoprì che gli anarchici milanesi non avevano nulla a che fare con l'attentato: i veri responsabili andavano cercati tra i militanti dell'estrema destra. Quella strage scosse il paese e l'opinione pubblica ne fu seriamente turbata, perché il livello di tensione sociale aveva raggiunto il limite sopportabile. 
Gli anni settanta iniziarono sulla scia provocata da tutti questi avvenimenti. Gli attentati proseguirono e si cominciò a parlare di «strategia della tensione», teoria secondo la quale una parte dei Servizi Segreti, i cosiddetti Servizi Segreti «deviati», si era infiltrata in alcuni gruppi terroristici di estrema destra e li aveva in qualche modo manipolati e spinti a commettere azioni tali da creare allarme sociale e giustificare così la necessità di uno Stato più autoritario. In una situazione tale, si radicalizzarono le proteste giovanili e operaie e si posero le fondamenta su cui si poggiò il gruppo terroristico italiano più tristemente famoso, le Brigate Rosse. La sua nascita si fa coincidere con l'estate del 1970, quando un gruppo di ragazzi appartenenti all'area marxista-leninista più radicale e provenienti da differenti zone d'Italia si riunì a Milano per dar vita a un'esperienza che cambierà le loro esistenze e quelle di molte altre persone. Per quasi vent'anni un manipolo di criminali insanguinò il paese con la pretesa di sovvertire lo Stato italiano e di imporre la rivoluzione popolare. Uno degli slogan più utilizzati in quell'epoca, non solo dai brigatisti ma anche dal loro brodo di coltura, era «instaurare la dittatura del proletariato», chiaro riferimento a Mao e alla sua rivoluzione culturale. Non ci riuscirono, ma solo per provarci, spezzarono più di ottanta vite.

I primi anni delle Brigate Rosse furono caratterizzati da azioni cosiddette di «propaganda armata», un eufemismo dietro il quale si celavano atti di sabotaggio all'interno delle fabbriche, distruzione di autovetture di propietà di capifabbrica e sequestri di persona. Questi ultimi vennero definiti express, dato che la loro durata si limitava a poche ore, passate le quali il malcapitato veniva liberato dopo aver subito un intenso interrogatorio sui temi riguardanti la sua attività all'interno della fabbrica in cui lavorava. Questa situazione perdurò fino al 1974, anno in cui le BR colpirono a morte per la prima volta. Le vittime erano due militanti del Movimento Sociale Italiano sorpresi all'interno della sede padovana del partito. I due vennero giustiziati con un colpo di pistola alla nuca. Passarono altri due anni prima che le Brigate Rosse ripetessero un'azione omicida. L'8 giugno 1976, a Genova, un commando terroristico uccise il giudice Francesco Coco e i due agenti di scorta. L'attentato dette inizio a una seconda fase, più cruenta, che prese il nome di «attacco al cuore dello Stato». A capo di questo nuovo momento criminale c'era Mario Moretti, leader militarista del gruppo, che aveva preso in mano le redini delle BR quando Renato Curcio e Alberto Franceschini, ideologi e fondatori dell'organizzazione, vennero arrestati dalla polizia l'8 settembre 1974. Dopo l'assassinio di Coco si iniziarono a vivere i momenti più terribili dell'attacco brigatista. Gli attentati e le esecuzioni si producevano con una frequenza disarmante. Giudici, avvocati, politici, giornalisti, poliziotti, carabinieri, capifabbrica, imprenditori, furono attaccati dalle Brigate Rosse.

L'avvenimento più noto anche fuori dall'Italia è, senza dubbio, il sequestro e il successivo assassinio di Aldo Moro, presidente della Democrazia Cristiana, partito che si mantenne al governo del Paese dal dopoguerra fino al crollo della prima Repubblica nei primi anni novanta. Le Brigate Rosse proseguirono nella loro assurda missione di morte fino al 1988 quando, finalmente, sembrava che anche l'ultimo degli irriducibili avesse abbandonato le armi. Oltre dieci anni dopo, purtroppo, il terrorismo collegato in qualche modo al brigatismo si ripresentò con tutta la sua violenza. Due consulenti del Ministro del Lavoro vennero freddati nel 1999 e nel 2002, 
così come un agente della Polfer nel 2003. Gli autori di questi delitti firmarono i loro volantini di rivendicazione con la stella a cinque punte e la scritta Brigate Rosse. Dopo uno sbandamento iniziale, durante il quale l'ombra del gruppo armato sembrava fosse riapparso ad oscurare la serenità, mai del tutto salda, della Repubblica italiana, si scoprì che, in realtà, queste nuove Brigate Rosse non avevano né la forza né tanto meno la coesione delle antiche a cui si ispiravano, tant'è che non si sono mai più fatte vive.

In numerosi casi, soprattutto nel passato, l'opinione pubblica ha faticato a interpretare la reale percezione di questo gruppo criminale, dato che la stampa italiana, specialmente nei primi anni del brigatismo, ha trattato il tema delle Brigate Rosse con colpevole disattenzione e superficialità.

In un primo momento sui giornali apparvero articoli in cui si sottovalutava l'effettiva potenza di questa organizzazione. Con il passar del tempo la percezione cambiò e si arrivò a una sconsiderata esaltazione della potenza criminale dei brigatisti che finirono per essere ritenuti dei «samurai invincibili», tanto per usare le parole di un celebre giornalista, Walter Tobagi, che venne assassinato da un gruppo terrorista di estrema sinistra ${ }^{2}$. Un ex brigatista, Alfredo Bonavita, ammise che il ruolo della stampa era stato fondamentale per aumentare in maniera spropositata la grandezza delle azioni del gruppo armato in cui militava ${ }^{3}$.

Colpevoli furono anche numerosi giornalisti, rappresentanti delle più importanti testate del paese, che si prodigarono nell'esercizio di sottrarre chiarezza a quanto chiaro sembrava un po' a tutti, tranne che a loro. Per molto tempo, troppo tempo, alcuni giornali, per non dire tutti, si impegnarono a considerare le Brigate Rosse una formazione armata di estrema destra, ignorando quanto gli stessi terroristi scrivevano nei loro comunicati rivendicativi in cui vincolavano le loro azioni alla causa marxista-leninista. In maniera più chiara era impossibile dirlo anche per loro, che nei loro scritti non erano certo accessibili, ma abusavano di un linguaggio criptico e fumoso; quando invece i brigatisti parlavano della loro ideologia erano chiari e diretti come non mai. In troppi purtroppo fecero finta di non capire. In questo articolo procederemo ad analizzare l'atteggiamento di gran parte della stampa italiana, almeno fino ai primi anni ottanta. A partire da questo periodo, infatti, da una parte cala l'intensità degli attacchi brigatisti, dall'altra la stampa inizia a informare in maniera più coerente e verosimile.

\section{IL POTERE DI UN'INFORMAZIONE}

Si è scritto molto sul rapporto che si crea tra terrorismo e mezzi di comunicazione, e ancor di più dopo alcuni avvenimenti che hanno scosso in maniera diretta il

\footnotetext{
2 Walter Tobagi, ex giornalista del Corriere della Sera, intitolò uno dei suoi ultimi articoli prima di morire «Non sono Samurai invincibili» riferendosi proprio all'eccessivo timore reverenziale che si stava creando in Italia nei confronti dei terroristi. Pagò con la vita il coraggio di aver pubblicato quelle parole.

3 Intervista a Alfredo Buonavita nel programa La notte della Repubblica condotto da Sergio Zavoli, Televisione Rai, 1989.
} 
mondo occidentale. Le tragedie dell'11 settembre 2001 a New York, dell'11 marzo 2004 a Madrid e del 7 luglio del 2005 a Londra hanno portato a riflettere più di uno studioso sulla diabolica connessione presente tra media e azioni terroristiche. Secondo quanto afferma Luís Veres esiste una simbiosi tra questi due fattori, dato che i terroristi trovano nei mezzi di comunicazione la maniera per dar eco alle proprie azioni e, allo stesso tempo, confezionano lo «spettacolo» di cui i giornalisti hanno bisogno per soddisfare i loro lettori (Veres 2002: 2). Non a caso quanto più orribile è l'attacco terroristico più grandi sono i titoli che appaiono sui giornali (Veres 2005: 594). Il successo di un'operazione terroristica si misura, così come afferma Laqueur, con la quantità di pubblicità che riceve, e sono gli stessi terroristi che cercano questa divulgazione perché sanno che senza diffusione l'attentato non avrebbe alcun senso. In questa maniera «non è la dimensione dell'azione terroristica che conta, ma il clamore che produce» e non sono pochi i casi in cui, proprio i mezzi di comunicazione, per colpa della loro innata ricerca del sensazionalismo, hanno «ingigantito le prodezze dei terroristi molto al di sopra della loro intrinseca importanza. Gruppi terroristici che avevano una dozzina di militanti sono stati descritti come se fossero eserciti» (Laqueur 1978: 145-146).

Il peso mediatico dopo un attentato è fondamentale per i terroristi; di fatto, sempre con l'aiuto di Veres, scopriamo che «la differenza tra un qualsiasi atto criminale e l'atto terroristico, al margine delle motivazioni politiche, si ritrova nel fatto che pochi delinquenti comuni non necessitano di pubblicità per soddisfare le proprie aspirazioni, mentre i terroristi esigono necessariamente questa propaganda affinché si compiano i loro fini nella loro totalità» (Veres 2005: 584).

Sono gli stessi brigatisti coloro i quali corroborano queste teorie. Uno dei fondatori del gruppo, Alberto Franceschini, per esempio, dichiarò in un'intervista rilasciata al giornalista Sergio Zavoli molti anni fa che per le Brigate Rosse i giornali sono stati importanti come punto di riferimento dato che, in fondo, l'azione terroristica si può considerare come una notizia preconfezionata (Zavoli 1992: 405).

Si inverte così il rapporto tra un fatto di cronaca e una notizia: se la norma ci suggerisce che il primo, in maniera fortuita, genera la seconda, nel caso di un attacco terroristico quest'ordine viene stravolto ed è l'avvenimento ad essere provocato affinché sia prodotta una notizia. Senza arrivare agli estremi di Umberto Eco che ritiene che «se non esistessero i mezzi di comunicazione, non si produrrebbero quegli atti destinati a essere notizia» (Eco 1977: 152), è allo stesso tempo possibile ritenere fondamentale la loro presenza per generare una pubblicità gratuita attorno ad un'azione armata.

Dopo questa breve disamina dovremmo interrogarci sull'opportunità o meno di fornire notizie vincolate al terrorismo, per non trasformare gli organi di informazione in casse di risonanza di una strategia criminale. Proprio durante l'attivismo armato delle Brigate Rosse, in Italia si dibattè molto sulla possibilità di «staccare la spina» e smettere di informare. In alcuni casi, come vedremo in seguito, si arrivò addirittura a realizzare una sorta di blackout, ma l'esperienza non fu positiva e non si ripetè mai più. $\mathrm{Fu}$ il sociologo canadese Marshall McLuhan colui che più si prodigò, nella seconda parte degli anni settanta, nel proselitismo della necessità di oscurare i mezzi di comunicazione per stroncare sul nascere qualsiasi possibilità di mani- 
polazione dell'informazione da parte dei terroristi. La proposta dello studioso, pubblicata sulle pagine del Corriere della Sera proprio durante il sequestro di Aldo Moro, tra il serio e la provocazione, riuscì a scuotere gli animi di molti giornalisti e produsse un intenso dibattito ${ }^{4}$. La proposta di McLuhan, naturalmente, non era facile da accettare, specialmente in un paese democratico in cui l'informazione è un diritto dei cittadini. Ángel Benito ci ricorda che «anche un attentato apparentemente minore risulta molto più efficace rispetto a un'operazione di grandi dimensioni se i mezzi di comunicazione se ne occupano in maniera profonda. È evidente che questa strategia può funzionare solamente in società in cui non esiste la censura» (Benito 1986: 124).

Come segnala Gabriel Sánchez Rodríguez, «fornire informazioni sul terrorismo è una missione difficile, ma necessaria» e aggiunge:

Non è possibile il silenzio informativo di fronte ad azioni terroristiche, il blackout. Affinché si produca questa circostanza sarebbe necessario un ferreo, esaustivo ed estremamente rigoroso controllo dell'informazione da parte di tutti i mezzi di comunicazione, situazione francamente difficile da immaginare [...] Potrebbe esserci silenzio anche nel caso in cui ci fossero meccanismi di restrizione della libertà d'espressione. In ognuno dei casi non sembra molto probabile il successo di questa precauzione. Né i professionisti dei mezzi di comunicazione né la società accetterebbero queste limitazioni [...] Inoltre, se si mette un bavaglio, oltre a non adempiere all'impegno sociale di informare, i mezzi di comunicazione incorrerebbero in un altro errore: la mancanza d'informazione e trasparenza sarebbe occupata immediatamente da rumori, mezze verità, esagerazioni, distorsioni della realtà e da tutto un cumulo di falsità che nascono e crescono attorno al fatto che si vuole nascondere (Sánchez Rodríguez 2005: 78).

In effetti nella società in cui viviamo, caratterizzata dal predominio quasi eccessivo dei mezzi di comunicazione che nella maggior parte dei casi interferiscono nella vita privata dei cittadini, decidere di sospendere l'informazione sarebbe impensabile, anche perché, al giorno d'oggi non ci confrontiamo unicamente con la carta stampata e la televisione, esistono moltissimi mezzi per informarsi ed informare. Una sospensione totale sarebbe una follia e, concordo con Sánchez Rodríguez, l'azione di occultamento di notizie potrebbe creare delle false informazioni molto facili da manipolare e che risulterebbero ancora più negative.

La necessità è piuttosto un'altra: quella di diffondere le notizie in una maniera seria, rispettando i canoni etici giornalistici tra i quali predomina la salvaguardia della verità. Come vedremo in queste pagine, durante gli anni settanta, attorno al terrorismo brigatista fu fatta una notevole confusione da parte di molti giornali. Valga un esempio per tutti, quello di Giorgio Bocca, prestigiosa firma di molti quotidiani italiani che nel 1975 sulle pagine de Il Giorno sostenne:

${ }^{4}$ Intervista a Marshal McLuhan, «Ridurre al minimo lo spazio ai terroristi», Corriere della Sera, 23-III1978. 
A me queste Brigate Rosse fanno un curioso effetto di favola per bambini scemi e insonnoliti; e quando i magistrati e gli ufficiali dei Carabinieri e i prefetti ricominciano a narrarla, mi viene un'ondata di tenerezza, perché la favola è vecchia, sgangherata, puerile, ma viene raccontata con tanta buona volontà che proprio non si sa come contraddirla ${ }^{5}$.

Va notato fin da subito che al momento di pubblicare questo articolo le Brigate Rosse avevano cinque anni di vita, avevano bruciato automobili, inviato comunicati minacciosi, sequestrato persone e ucciso due militanti del Movimento Sociale Italiano. Va anche detto che Bocca, molti anni dopo, riconobbe il suo errore e chiese scusa pubblicamente.

\section{PICCOLI E GRANDI ERRORI QUOTIDIANI}

L'errore in cui era incappato uno dei più noti giornalisti italiani venne ripetuto in maniera costante da molti altri suoi colleghi per buona parte degli anni settanta. In pochi hanno provato ad addentrarsi nei meandri della stampa italiana per analizzare il rapporto ambiguo che questa mantenne con le Brigate Rosse. Michele Brambilla fu uno di quelli e il suo lavoro, serio e meticoloso, è fondamentale per chi oggi si cimenta in questa ardua ricerca (Brambilla 1991).

La prima notizia che apparve sui giornali italiani inerente alle Brigate Rosse risale al 26 gennaio 1971, quando il Corriere della Sera informò di un atto di sabotaggio verificatosi nella pista-prove della Pirelli nella sede di Lainate. Si parla di «Brigata Rossa» così come riportato sul volantino rivendicativo e come gli stessi brigatisti erano soliti firmare i loro primi comunicati, al singolare ${ }^{6}$. La banda terroristica era nata già da qualche mese, però fino a quel momento aveva richiamato l'attenzione di poche persone. Il lancio di bombe incendiarie a Lainate fu il primo atto di guerriglia che i brigatisti ritennero propagandisticamente positivo. Fu così che si iniziò lentamente a parlare di questo gruppo di cui non si sapeva nulla, tanto meno ciò che volevano ottenere con l'utilizzo della violenza. Già in questo primo articolo, apparve l'aggettivo «fantomatico» accanto a Brigate Rosse, uno degli epiteti che più saranno utilizzati per definire, in tono sarcastico, l'organizzazione. Le BR erano già dall'inizio considerate fantomatiche, sedicenti, immaginarie, irreali, favolose, fantasmagoriche, chimeriche, tutti sinonimi che avevano l'unico obiettivo di moderare e smussare la reale capacità di attacco di questo gruppo. È necessario dire, per cercare di scusare in qualche modo la disattenzione della stampa nei suoi primi momenti, che in quel periodo l'Italia aveva problemi ben più seri da fronteggiare. D'altra parte, il gruppo di Curcio si era appena costituito e le sue prime azioni non differivano molto dagli attacchi provocati dalla protesta operaia

\footnotetext{
5 Giorgio Bocca, «L'eterna favola delle Brigate Rosse», Il Giorno 2-II-1975.

${ }^{6}$ All'inizio degli anni settanta esisteva solamente una brigata, proprio quella che operava alla Pirelli.
} 
più radicale ${ }^{7}$. Il fatto più preoccupante è che per quasi tutti gli anni settanta il gruppo terroristico, sui giornali, continuava ad apparire fantomatico, anche dopo numerosi omicidi.

I terroristi iniziarono a mettere in pratica i sequestri express e la stampa italiana, anche di fronte a un aumento sensibile dell'impatto criminale, non cambiò il suo atteggiamento accondiscendente e dubitativo. Il 4 marzo 1972 L'Unità, organo di informazione del Partito Comunista Italiano, definì quanto accaduto a Idalgo Macchiarini, responsabile del personale della Sit-Siemens di Milano, rapito per alcune ore da un gruppo di militanti brigatisti incappucciati, una «banditesca provocazione antioperaia». Il quotidiano dichiarò, inoltre, che il «fantomatico gruppo era di chiara ispirazione fascista $\rangle^{8}$. Non solo si cercava di stemperare un atto criminale decisamente grave come il sequestro di una persona, ma, senza alcun fondamento, si etichettava quanto accaduto come opera di esponenti neofascisti ignorando la rivendicazione in chiave marxista-leninista fatta dagli stessi autori dell'attaco.

$\mathrm{Fu}$ inquietante un articolo che apparve, sempre su L'Unità, pochi giorni dopo. Sotto il titolo «Qualcosa di americano» si analizzava la foto che ritraeva Macchiarini durante il suo sequestro; fotografia inviata ai principali quotidiani del paese proprio dai brigatisti. Nell'immagine, tra le più note della storia delle Brigate Rosse, si scorge una pistola puntata alla tempia dell'ingegnere che sorregge un grande cartello colmo di scritte rivoluzionare. Ebbene questa fu l'analisi che venne fatta dell'istantanea: «Il dirigente industriale rapito o è un uomo di sublime coraggio, o è un incosciente, o è un pessimo attore che non riesce a recitare la parte affidatagli. Infatti egli se ne sta sotto la minaccia ravvicinata delle pistole senza mostrare alcun segno, non diciamo di paura o di smarrimento, ma neppure di preoccupazione» ${ }^{9}$.

Se L'Unità non fosse una testata che all'epoca vendeva circa 240 mila copie al giorno, questo intervento parrebbe semplicemente ridicolo e probabilmente accuseremmo di poca esperienza il giornalista che lo ha scritto, invece stiamo parlando di uno dei giornali più letti nell'Italia degli anni settanta, dottrina quasi assoluta per molti militanti comunisti di quell'epoca. Un'analisi di questo tipo era certamente fuorviante e pericolosa, oltre che del tutto falsa e poco benevola nei confronti di chi aveva subito un grave oltraggio alla propria persona e alla propria libertà. L'articolo proseguiva con un pensiero ancora una volta fuorviante secondo il quale si dava per scontato che le Brigate Rosse fossero in realtà «brigate nere», rinconducibili quindi all'area neofascista. È grave osservare la facilità con cui un giornalista, di fronte a una rivendicazione fatta dagli autori del rapimento, si prenda la libertà di sconfessarli e di interpretare quanto da loro detto in chiave totalmente opposta.

Nel mese di maggio di quello stesso anno 1972, la polizia sferrò il primo grave colpo ai danni dell'organizzazione fino a quel momento quando, all'interno di un

\footnotetext{
7 Non va dimenticato che in Italia era iniziata la cosiddetta «strategia della tensione», una trama occulta di attentati in cui parteciparono, come si dimostrerà in seguito, settori «deviati» del potere. Continuavano anche le proteste studentesche e operaie e l'economia stava per subire dei duri colpi alla sua stabilità.

8 «Vile aggressione ad un dirigente della Sit-Siemens», L'Unità, 4-III-1972.

9 «Qualcosa di americano», L'Unità, 15-III-1972.
} 
appartamento di Milano, trovò un arsenale brigatista formato da volantini, documenti falsi, pistole, munizioni, bombe, esplosivo. Il Corriere della Sera dedicò un ampio spazio al ritrovamento e il giornalista Giorgio Zicari utilizzò per la prima volta il termine «terrorista» per definire la banda criminale. Zicari nel suo pezzo sottolineava che, data l'ingente quantità di materiale sequestrato, era facile presumere che ci si trovava di fronte a un «ampio progetto terrorista ${ }^{10}$. E chiaro che, dopo una tale scoperta non aveva più senso proseguire con il sarcasmo sulle «fantomatiche Brigate Rosse», quando era più che chiaro che quei criminali avevano tutte le carte in regola per commettere attentati. Tuttavia L'Unità continuava a utilizzare un tono irrisorio, parlava di «sedicenti Brigate Rosse» (altro termine molto utilizzato in quel periodo) e di «organizzazione di provocatori» che agisce «al servizio di loschi interessi nel quadro della strategia della tensione» ${ }^{11}$, vale a dire, un gruppo evidentemente riconducibile al neofascismo.

I sequestri continuarono. Il 14 febbraio 1973 un gruppo di brigatisti rapì per alcune ore Bruno Labate, sindacalista giallo della CISNAL di Torino. La città della FIAT subiva così il suo battesimo da parte del terrorismo e fu uno dei centri più colpiti dalla violenza delle Brigate Rosse. Ancora una volta sui giornali si tentò di mitigare quanto accaduto e così il quotidiano milanese Il Giorno si limitò a parlare di «incredibile episodio di vandalismo» ${ }^{12}$; secondo L'Unità continuava a trattarsi di «gravi provocazioni» ${ }^{13}$; Il Manifesto fu ancora più diretto asserendo che quanto accaduto non era altro che un montaggio organizzato dal sindacato di destra. Nessuno ammetteva che si trattava, per lo meno, di un atto criminale.

Quando, pochi mesi dopo, le BR rapirono a Milano il vicedirettore dell'Alfa Romeo Michele Mincuzzi, L'Unità dichiarò senza esitazione che si trattava dell'ennesima azione firmata dalle «sedicenti Brigate Rosse, dietro la cui etichetta si celano elementi provocatori che agiscono nell'interesse di chi vuole alimentare la strategia della tensione» ${ }^{14}$. Tutto come prima, quindi, la linea editoriale dell'organo d'informazione del Partito Comunista Italiano non si spostava dalle posizioni precedenti. Le BR continuavano ad essere un «gruppuscolo di provocatori agli ordini di strategie ben più complesse e serie». Il Corriere della Sera dette ampio spazio alla notizia del sequestro di Mincuzzi, però risulta strana la conclusione a cui arrivò Giovanni Moncini, il quale dichiarò che «non è nostro compito dire quale sia il colore di questa impresa». Forse Moncini aveva ragione, però quasi tutti i giornali si sbizzarrirono nel trovare una collocazione a destra alle Brigate Rosse. Un esempio chiaro è quello del suo collega Livio Esposito che si domandava se si trattasse realmente di un «gruppo esasperato dell'estrema sinistra» o fosse piuttosto «un gruppo dell'estrema destra anti-maoista o fascista camuffato» ${ }^{15}$. Alberto Sensini, anch'egli

${ }^{10}$ Giorgio Zicari, «Una prigione del popolo in una cantina di Milano», Corriere della Sera, 3-V-1972.

${ }^{11}$ Ibio Paolucci, «La polizia scopre due arsenali a Milano con corredo di fotografie e passaporti», $L$ ' $U$ nità, 3-V-1972.

12 Marco Nozza, «Sindacalista del MSI sequestrato a Torino», Il Giorno, 13-II-1973.

13 «Gravi provocazioni a Torino», L'Unità, 14-II-1973.

14 «Ferito e sequestrato per tre ore a Milano un vicedirettore dell'Alfa Romeo», L'Unità, 29-VI-1973

${ }^{15}$ Livio Esposito, «Fanatici della guerriglia urbana», Corriere della Sera, 11-XII-1973. 
dalle colonne del quotidiano milanese, il giorno successivo rincarava la dose: «Queste fantomatiche Brigate Rosse che vorrebbero apparire di estrema sinistra ma fanno sistematicamente cose che giovano solo all'estrema destra, accusano tutti di fascismo ma praticano il peggior fascismo che si veda in Italia dopo la caduta di Salò, parlano di lotta di classe ma danneggiano soltanto chi lavora ${ }^{16}$.

Il 10 dicembre 1973 fu sequestrato a Torino Ettore Amerio, capo del personale della FIAT Mirafiori: si trattava del primo rapimento brigatista che durò per oltre una settimana, discostandosi dal «mordi e fuggi» a cui ci avevano abituato le Brigate Rosse fino a quel momento. Nonostante l'estensione dell'azione criminale, la campagna di disinformazione continuava. Quando la polizia identificò uno dei possibili rapitori, questa è la descrizione che ne fece Enzo Passanisi sul Corriere della Sera: «Massimo riserbo sulla collocazione e sull'estrazione del gruppo: si è saputo soltanto che l' «ardito» [così si faceva chiamare il brigatista arrestato] e i suoi seguaci si staccarono da un gruppuscolo dell'estrema sinistra per costituire un movimento autonomo». Fin qui nulla di anomalo, però più avanti leggiamo: «Movimento in cui, secondo uno schema ormai consueto nelle frange eversive, sarebbero confluiti provocatori della parte opposta, anime $\langle$ nere $»\rangle{ }^{17}$.

Questa seconda parte non è affato documentata. È del tutto superflua, però viene aggiunta come a giustificare che un gruppo marxista-leninista difficilmente si abbandonerebbe a simili pratiche banditesche senza l'intromissione di frange neofasciste.

L'Unità analizzò quanto accaduto a Ettore Amerio in una maniera ancor più inquietante. Diego Novelli, futuro sindaco di Torino e prestigiosa «penna» del giornale fondato da Gramsci, in un lungo articolo apparso il 12 dicembre riproponeva uno scenario quanto meno fantasioso. Per capire a fondo l'inconsistenza di quanto riportato da Novelli, bisogna fare un passo indietro per scoprire che, secondo alcuni testimoni, i rapitori agirono travestiti da tecnici della compagnia telefonica SIP. Ebbene, questo è quanto riportato dal giornalista:

Oggi abbiamo avuto modo di accertare, sulla base di una testimonianza più che attendibile, la veridicità di un episodio accaduto alcuni mesi orsono in un'azienda metalmeccanica della Valle di Lanzo. Una squadra di operai incaricati della manutenzione telefonica presso questa ditta si era presentata in abbigliamento piuttosto sconcertante: sotto le tute gli incaricati della società indossavano maglioni neri con al collo medaglioni fascisti e croci uncinate ${ }^{18}$.

Come si può vedere, l'articolo si basa su illazioni e su di una presunta testimonianza non documentata. Il nesso tra un rapimento alla FIAT e un manipolo di tec-

16 Salò si riferisce alla Repubblica Sociale, governo fascista formato da Mussolini nel 1943 e con sede in questa piccola località sul lago di Garda, Salò appunto. L'articolo citato è di Alberto Sensini, «Strappare la spoletta», Corriere della Sera, 12-XII-1973.

17 Enzo Passanisi, «Identificati i rapitori di Ettore Amerio», Corriere della Sera, 16-XII-1973.

18 Diego Novelli, «Si delineano i torbidi retroscena sul rapimento del dirigente FIAT», L'Unità, 12-XII1973. 
nici vestiti in maniera poco consona a un ambiente di lavoro in una ditta delle colline piemontesi non regge. Ebbene, queste forzature erano all'ordine del giorno in quel periodo. Il Manifesto fu ancora più diretto e scrisse che sotto la denominazione di Brigate Rosse si nascondeva una delle tante bande di fascisti da anni impegnate nella provocazione ai danni della classe operaia ${ }^{19}$.

Stessa linea editoriale per Il Giorno, che si chiedeva se i criminali fossero «brigatisti rossi o giustizieri neri» mettendo in dubbio, ancora una volta, la reale ideologia dell'organizzazione.

Si arrivò così al 18 aprile 1974, giorno in cui le Brigate Rosse rapirono il magistrato del tribunale di Genova Mario Sossi, Pubblico Ministero nel processo alla banda terrorista di estrema sinistra XXII Ottobre. Un gruppo di venti brigatisti lo sequestrò e lo sottopose a interrogatorio per oltre un mese prima di liberarlo. Questa azione criminale si produceva proprio a ridosso di un'importante consultazione popolare, il referendum attraverso il quale, poco meno di un mese più tardi, gli italiani avrebbero avuto la possibilità di abrogare la legge che avrebbe reso operativo il divorzio. Le forze politiche dell'epoca avevano trasformato il plebiscito in una specie di termometro sociale che poteva dare dei chiari segnali sul futuro istituzionale del paese. Da una parte vi erano la Democrazia Cristiana e le forze di destra che osteggiavano quella legge, dall'altra i partiti progressisti che appoggiavano la riforma. Per i quotidiani fu molto semplice collegare il referendum all'azione delle Brigate Rosse. Il Corriere della Sera parlava nuovamente di «strategia della tensione che sistematicamente colpisce il nostro paese alla vigilia di importanti consultazioni politiche e quando il clima è più agitato ${ }^{20}$. L'Unità sottolineava il momento in cui si trovava l'Italia e diceva che «ancora una volta in un momento tra i più delicati, la vita del Paese viene turbata profondamente da un episodio criminale di provocazione», facendo un chiaro riferimento al giudizio popolare di maggio ${ }^{21}$. Ibio Paolucci, lo stesso giorno in quinta pagina, sottolineava che «le Brigate Rosse attaccano quando serve alla destra» e che «l'ultima impresa criminale delle sedicenti Brigate Rosse ha spazzato definitivamente ogni dubbio sulla loro reale natura», riferendosi chiaramente alla destra reazionaria ${ }^{22}$. Il socialista Avanti!, oltre a posizionarsi sulla falsariga dei giornali sin qui analizzati affermando che i brigatisti appaiono «nei momenti più cruciali della vita politica e sociale del Paese», parlava di «criminali travestiti di rosso $»^{23}$ ed affermava che tra gli adepti delle BR vi fossero «elementi che presumono di essere di sinistra $»^{24}$, ma che di sinistra in realtà non sono. Poche novità quindi, però la colpevolezza di continuare, dopo quattro anni dalla nascita delle BR, a mantenersi sulle stesse posizioni oltranziste di negazione della vera natura del gruppo terroristico.

19 «Per il rapimento del dirigente FIAT torna il nome del provocatore Pisetta», Il Manifesto, 15-XII1973.

20 Alberto Sensini, «Un'altra sfida allo Stato», Corriere della Sera, 20-IV-1974.

21 «Una trama evidente», L'Unità, 20-IV-1974.

22 Ibo Paolucci, «Le Brigate Rosse colpiscono quando giova alla destra», L'Unità, 20-IV-1974.

${ }^{23}$ Roberto Presenti, «Le Brigate del caos», Avanti!, 20-IV-1974.

24 «Chi sono e per conto di chi?», Avanti!, 20-IV-1974. 
Pochi giorni dopo, un articolo apparso sul Corriere della Sera sembrava finalmente modificare il monotono giudizio che considerava le BR un gruppo reazionario. Si trattava di un'intervista a un esperto di terrorismo il cui nome non veniva rivelato per non esporlo ad un inutile pericolo. Lo studioso affermava con chiarezza che chi aveva sequestrato Sossi era un'organizzazione in cui militavano «sociologi dell'Università di Trento», «il Collettivo Politico Metropolitano, più tardi trasformatosi in Sinistra Proletaria, alcuni marxisti-leninisti e un paio di anarchici» ${ }^{25}$. Il giornalista non sembrava però molto convinto delle parole dell'esperto e così gli chiedeva reiteratamente se fosse realmente sicuro di quanto diceva. La risposta non lasciava nessun dubbio: «la realtà è questa. La sua connotazione politica, per lo meno formalmente è indiscutibile». Ma diceva anche altro:

È vero, molte volte queste Brigate Rosse fanno pensare a fascisti che si mascherano da comunisti per creare problemi alla sinistra italiana. Però è anche vero che ricevono la solidarietà di alcuni gruppi della sinistra extraparlamentare, che pubblicano nelle loro riviste i documenti delle Brigate Rosse, e polemizzano, anche in maniera molto dura, con voi giornalisti che definite le Brigate Rosse di colore neutro o addirittura nero ${ }^{26}$.

Quanto detto dall'intervistato fa gran luce su di un concetto che non è stato mai preso in considerazione dalla maggior parte della stampa italiana di quegli anni: l'atteggiamento del brodo di coltivo che appoggiava le azioni delle Brigate Rosse e che proveniva in maniera indiscutibile da ambienti di estrema sinistra. I gruppi extraparlamentari erano numerosi e attiravano un gran numero di giovani e meno giovani. Molte organizzazioni di quell'area ideologica pubblicavano riviste o giornali in cui, spesso, trovavano spazio i documenti delle Brigate Rosse. Cercare di occultare questi dati non aiutava certamente a fare chiarezza su quanto stesse succedendo allora.

Un altro punto estremamente controverso della faccenda Sossi è l'immagine che alcuni giornali diedero della vittima. Gli aggettivi più utilizzati per descrivere il magistrato rapito erano «duro», «intransigente», «rigoroso». Si parlava anche della sua vicinanza politica alla destra e dei suoi processi contro i gruppi dell'estrema sinistra, specialmente il già menzionato gruppo XXII Ottobre. Sembrerebbe, leggendo buona parte degli articoli di quei giorni, che alcuni giornalisti avessero fatto di tutto per giustificare l'azione criminale delle Brigate Rosse assolvendole proprio perché la vittima in qualche modo «se l'era cercata».

Di lì a pochi mesi, i due massimi dirigenti delle Brigate Rosse, Renato Curcio e Alberto Franceschini, vennero arrestati. Poco più di quattro mesi dopo, nel febbraio del 1975, un commando terrorista riuscì a liberare uno dei due, l'ideologo Renato Curcio, grazie a una spettacolare irruzione nel carcere di Casale Monferrato in cui si trovava. Il Corriere ridusse l'assalto a una prigione italiana a una semplice

${ }^{25}$ Il Collettivo Politico Metropolitano e Sinistra Proletaria erano due gruppi che anticiparono l'esperienza delle Brigate Rosse.

${ }^{26}$ «Identikit delle Brigate Rosse», Corriere della Sera, 24-IV-1974. 
«beffa ${ }^{27}$. I quotidiani di sinistra imputavano la fuga del terrorista all'intervento di gruppi armati di estrema destra o di qualche servizio segreto straniero.

Nel frattempo era apparso un nuovo quotidiano denominato Il Giornale Nuovo e diretto da uno dei più prestigiosi giornalisti italiani, Indro Montanelli, che insieme a un gruppo di colleghi aveva abbandonato il Corriere della Sera con cui era in rotta da quando la linea editoriale si era posizionata sempre più a sinistra. Questo nuovo organo di informazione dava voce alla borghesia milanese di stampo liberale che si era sentita un po' tradita dall'atteggiamento del Corriere. Furono proprio le colonne di questa nuova testata che più si prodigarono nel fare luce sulla vera natura delle $\mathrm{BR}$, a volte in maniera forse troppo sbrigativa e rigida. Il Giornale Nuovo si trovò presto isolato nel suo tentativo di smascherare chi si celava dietro la sigla rivoluzionaria brigatista.

Dobbiamo comunque attendere fino al 17 maggio del 1975, due giorni dopo l'attacco brigatista ai danni dell'esponente democristiano Massimo De Carolis, per trovare sulla stampa italiana un articolo che facesse un po' di chiarezza su quanto stava succedendo in quel periodo. Il suo autore era proprio Indro Montanelli, il quale dichiarava:

Non siamo affatto stupiti di quanto gli è capitato [a De Carolis]. Quanto è capitato a lui può ormai capitare a chiunque [...] Non vogliamo riprendere la polemica, tipicamente italiana nella sua vacuità, fra «opposti estremismi» e «strategia della tensione». Queste battaglie nominalistiche le lasciamo volentieri ai professionisti delle parole: nel nostro Paese ce ne sono anche troppi. Vediamo le cose nei fatti, come si stanno sviluppando sotto i nostri occhi e purtroppo sulla nostra pelle.

Si partì con un'affermazione di principio: la violenza viene sempre da destra perché la sua matrice è lì. Pure se non in termini così assoluti, l'asserzione ha una sua validità sul piano storico, e per un certo tempo ha trovato conferma anche nella cronaca [...] Quando dalla parte opposta cominciò a delinearsi un'altra violenza, non trovammo molti argomenti da contrapporre a chi la spiegava e giustificava come una reazione, sia pur disordinata, sia pur rozza e malaccorata, alla provocazione fascista. Nell'assenza dei pubblici poteri, nella paralisi sempre più accentuata dei servizi d'ordine, poteva avere una sua logica - anche se si tratta di una logica da jungla - l'intimidazione preventiva contro un'altra intimidazione, il deterrente di un terrorismo contrapposto a un altro terrorismo.

Ma questa fase appartiene ormai al passato. Di estremisti di destra ce ne sono certamente ancora (e se si continua di questo passo, temiamo che un giorno ce ne saranno molti di più), e noi siamo per primi convinti che la loro minaccia non sia da sottovalutare: come bombaroli hanno la mano e la strage facili. Ma il panorama che si apre al nostro sguardo, la sopraffazione sistematica, la vigliacca aggressione di gruppo contro l'individuo isolato, il sequestro di persona praticato su scala industriale come mezzo di finanziamento dell'azione terroristica, le irruzioni a mano armata nelle abitazioni private, il sudario di paura che al calar del sole avviluppa le strade delle nostre città, e specialmente di Milano, come ai tempi del coprifuoco: tutto ciò non ha più nulla a che fare con la destra e fa parte di un

27 Alberto Sensini, «Lo Stato umiliato», Corriere della Sera, 20-II-1975. 
nuovo capitolo che bisogna deciderci ad affrontare al di fuori dei soliti schemi. Quella a cui stiamo assistendo è una escalation alla distruzione non più della destra, ma del sistema, alla quale la «provocazione fascista» serve solo da alibi, ma un alibi sempre più logoro e meno credibile ${ }^{28}$.

Ho voluto riprodurre un'abbondante porzione dell' articolo perché mi sembra che finalmente qualcuno avesse aperto gli occhi. Purtroppo era l'unico. L'Unità ribadiva il concetto di «trama terrorista fascista», però per lo meno, in un articolo dall'emblematico titolo «Da che parte stanno?», si parlava di «commandos criminali ben armati e addestrati ${ }^{29}$. Per la prima volta l'organo di stampa del PCI ammetteva che i terroristi erano ben preparati e si discostava dalla pericolosa versione che vedeva questi militanti come dei ragazzotti strambi e disorganizzati.

Da parte sua, il Corriere della Sera presentò la notizia del ferimento di De Carolis eliminando le ormai famose aggettivazioni «fantomatica» e "sedicente» accanto al nome dell'organizzazione delle Brigate Rosse. La consapevolezza che effettivamente l'organizzazione iniziava a far sempre più paura si manifestò all'indomani dell'omicidio del giudice Francesco Coco, freddato nelle viuzze di Genova insieme ai suoi due uomini di scorta l' 8 giugno 1976. Il quotidiano della città ligure, Secolo $X I X$, parlava di «terroristi organizzati», chiarendo che «i pazzi, gli isolati, $i$ terroristi improvvisati non avrebbero potuto attaccare con tanta freddezza e lucidità» ${ }^{30}$. Ci fu comunque chi non fece giustizia alla vittima e, come Miriam Mafai dalle colonne del neonato giornale La Repubblica, definì Coco «fedele e inquietante servitore dello Stato», che «aveva della giustizia, della legge, dello Stato una concezione così scopertamente reazionaria da apparire quasi un caso da manuale» ${ }^{31}$. Una volta ancora, c'è chi prova a stemperare la durezza dell'atto criminale discreditando la vittima.

\section{ATTACCO ALLA STAMPA ITALIANA}

Dopo l'omicidio Coco le Brigate Rosse non smisero più di uccidere, seppellirono il paese sotto una spessa coltre di paura e terrore. Le azioni violente si susseguirono per anni e la reazione dei mezzi d'informazione iniziò a mutare; i terroristi se ne accorsero e decisero di punirli. Nel giro di tre giorni, tra l'uno e il tre giugno del 1977, vennero gravemente feriti Vittorio Bruno, direttore de Il Secolo XIX, Indro Montanelli, direttore de Il Giornale Nuovo ed Emilio Rossi, direttore del TG1. Le reazioni dei colleghi non si fecero attendere e si versarono fiumi d'inchiostro nel cercare di spiegare quanto accaduto, giudicarlo ed esprimere solidarietà ai malcapitati. Leggermente fuori dalle righe solamente il Corriere della Sera, in cui Indro Montanelli aveva lavorato per anni e che in occasione del suo ferimento non rende-

\footnotetext{
28 Indro Montanelli, «Attacco al sistema», Il Giornale Nuovo, 17-V-1975.

29 «Da che parte stanno», L'Unità, 16-V-1975.

30 Vittorio Bruno, «Terrorismo organizzato», Il Secolo XIX, 9-VI-1976.

31 Miriam Mafai, «Un fedele e inquietante servitore dello Stato», La Repubblica, 9-VI-1976.
} 
va nessun onore al suo vecchio collaboratore. All'indomani dell'attentato, il titolo in prima pagina si limitava a uno sterile «I giornalisti nuovo bersaglio della violenza», senza nemmeno citare il nome del collega ferito ${ }^{32}$. Poi in altri articoli, si dimostrava solidarietà con il malcapitato sottolineando però le divergenze ideologiche tra la linea editoriale del Corriere della Sera e quella de Il Giornale Nuovo. Una volta messi sotto pressione i giornalisti, la loro reazione fu radicalmente opposta a quella a cui ci avevano abituato da anni. All'improvviso, come per magia, le Brigate Rosse iniziarono a fare paura, ad essere organizzate, temibili, ben armate, coraggiose, terroriste. Non più una specie di fantasmi che attaccavano quando faceva comodo alle forze reazionarie. Ranieri Orlando sul Corriere della Sera sembrava rassegnato nel suo articolo di fondo dall'emblematico titolo «Il nostro turno» ${ }^{33}$, L'Unità finalmente parlava di «terrorismo» ${ }^{34}$ e dichiarava che era in pericolo tutta la classe giornalistica italiana indipendentemente dall'ideologia politica professata ${ }^{35}$, anche $L a$ Repubblica non aveva più imbarazzi nel definire i brigatisti «terroristi» ${ }^{36}$, il Corriere della Sera proponeva «nuove leggi» nel caso in cui quelle vigenti non fossero sufficienti per frenare la violenza ${ }^{37}$. L'allarmismo cresceva senza tregua e così c'era chi, come Ulderico Munzi, dalle pagine del Corriere della Sera dichiarava che i brigatisti colpivano e fuggivano come e dove volevano ${ }^{38}$. In definitiva con l'attacco alla stampa italiana i criminali ottennero un surplus di notorietà e un ingigantimento delle loro effettive forze. È pur vero che in quell'epoca le Brigate Rosse si stavano preparando al loro colpo più tristemente brillante: il rapimento e il successivo assassinio del presidente della Democrazia Cristiana Aldo Moro. Non bisogna nemmeno dimenticare che il potere dei brigatisti venne ingigantito dal fatto che riuscirono ad attaccare dalla clandestinità più assoluta e anche per alcune colpe organizzative delle forze di sicurezza italiane che tardarono prima di reagire in maniera efficace.

Pochi mesi dopo il triplice ferimento avvenuto nei primi giorni di giugno, balzò alle cronache un nuovo assalto a un giornalista. Si trattava di Carlo Casalegno, cronista de La Stampa di Torino, città sommersa dalla violenza eversiva nella seconda metà degli anni settanta. Questa volta, a differenza di quanto avvenuto poco prima dell'estate, la vittima perse la vita sotto i colpi dei suoi carnefici. La condanna di questo gesto criminale fu unanime e fu seguita dalla consapevolezza che i terroristi erano passati dal proiettile nelle gambe alla pallottola in testa. Tutta la categoria si sentiva minacciata e la solidarietà al «servo dello Stato», così come definirono la loro vittima i brigatisti nel comunicato di rivendicazione, fu totale. Le parole più emotive vennero scritte dal direttore del giornale in cui Casalegno lavorava: «le Bri-

32 «I giornalisti nuovo bersaglio della violenza. Le Brigate Rosse rivendicano l'attentato», Corriere della Sera, 3-VI-1977.

${ }^{33}$ Ranieri Orlando, «Il nostro turno», Corriere della Sera, 3-VI-1977.

34 «Il calcolo del terrorismo», L'Unità, 3-VI-1977.

35 «Qual è il bersaglio?», L'Unità, 4-VI-1977.

36 «Attentato a Montanelli», La Repubblica, 3-VI-1977.

37 «Una difesa c'è», Corriere della Sera, 5-VI-1977.

38 Ulderico Munzi, «L'arma segreta dei terroristi», Corriere della Sera, 5-VI-1977. 
gate Rosse lo hanno definito «servo dello Stato». Non credo che io, i suoi colleghi, i suoi lettori potessero trovare una definizione migliore. Lui è un fedele, onesto e coraggioso servo dello Stato democratico» ${ }^{39}$.

Enzo Biagi, prestigiosa firma del Corriere della Sera, scriveva che non considerava un'infamia essere definito "servo dello Stato» e avvertiva i brigatisti che la loro rivoluzione non aveva nessun proselitismo ${ }^{40}$. In realtà una porzione di appoggio, seppur limitata, le Brigate Rosse l'avevano e non solo tra gli ambienti giovanili più radicali, ma anche dentro alle fabbriche, tra quegli operai che i terroristi stessi volevano guidare alla rivolta. Le BR incutevano sempre più terrore e la campagna di attacco ai giornalisti fece sì che si modificasse in maniera risolutiva quella titubanza che si era andata creando negli anni anteriori e che considerava la banda criminale come un manipolo di disadattati e di teppisti. Dal teppismo si era passati con sorprendente velocità al terrorismo, all'organizzazione militare, al pericolo pubblico. Ma l'azione più clamorosa di quegli assassini doveva ancora compiersi.

\section{COME VENNE TRATTATO DALLA STAMPA ITALIANA IL SEQUESTRO DI ALDO MORO}

Senza alcun dubbio l'azione più tristemente nota delle Brigate Rosse fu il sequestro e il successivo assassinio, quasi due mesi dopo, del presidente della Democrazia Cristiana, Aldo Moro. Era il 16 marzo del 1978 quando a Roma un commando terrorista, con un'imboscata militarmente perfetta, bloccò l'auto in cui viaggiava il politico con due uomini della scorta e il veicolo di rinforzo in cui vi erano altri tre militari. In poco meno di cinque minuti i brigatisti annientarono gli agenti e prelevarono Aldo Moro incolume. In tutta Italia iniziò una caccia ai banditi che durò per cinquantacinque giorni e si concluse in maniera tragica. Il 9 maggio, il corpo senza vita dello statista venne ritrovato nel baule di una Renault 4 di color rosso parcheggiata nel pieno centro della capitale. Si concludeva così la beffa più grande mai mossa contro lo Stato italiano. Ma proviamo ad analizzare in che modo i giornali di quell'epoca informarono sulle sorti del prigioniero. Si è già visto come la stampa italiana da qualche mese aveva abbandonato la diffidenza sull'effettiva pericolosità dell'organizzazione, specialmente quando i terroristi iniziarono a colpire proprio i giornalisti. Dopo il sequestro di uno dei politici più famosi d'Italia era evidente che le reazioni fossero ancor più allarmanti.

Analizzando l'evoluzione informativa durante gli oltre cinquanta giorni di sequestro, è interessante notare come, dopo un iniziale bombardamento di notizie, tutto si calmò e si faticò a consegnare al lettore delle novità su uno dei fatti di cronaca più sconvolgenti della storia della Repubblica italiana. Nella «prigione del popolo», il luogo in cui Moro era mantenuto ingabbiato e in cui i brigatisti lo inter-

39 «Casalegno in fin di vita», La Stampa, 17-XI-1977.

40 Enzo Biagi, «Che guerra è la loro?», Corriere della Sera, 17-XI-1977. 
rogarono fino al giorno dell'esecuzione, si viveva una sorda negoziazione tra la vittima, i suoi carnefici e i dirigenti della Democrazia Cristiana. Sin dai primi giorni iniziò un intenso carteggio tra le tre parti. I terroristi diffusero con scadenza costante i loro comunicati rivendicativi in cui si riportavano parti dell'interrogatorio del prigioniero. Da parte sua Aldo Moro si prodigava nella stesura di intense lettere indirizzate alla sua famiglia (brevi, ma emotive) e ai colleghi di partito (lunghe, criptiche e, in alcuni casi, disperate e irate) ma la DC rispondeva abbracciando la linea della fermezza. Sin da subito i carnefici commisero un errore fatale che condizionò tutte le trattative: insieme a uno dei loro primi comunicati diffusero pubblicamente anche una lettera di Aldo Moro. I giornali approfittarono il succulento regalo e non esitarono a pubblicare entrambe le missive. Gli uomini della Democrazia Cristiana, gran parte della classe politica italiana, molti opinionisti, adottarono la strategia di declassare le parole dal presidente giacché scritte in una situazione troppo estrema, che sottraeva ogni credibilità a quanto detto. In poco tempo, i mezzi d'informazione italiani trasformarono Aldo Moro da potente statista in grado per molti anni di governare il Paese, a disperato personaggio da tragedia il cui destino era già segnato e le cui parole non valevano nulla in quanto pronunciate in una circostanza e in un luogo che sicuramente avevano minato la sua integrità fisica e mentale. Da uomo potente a uomo sull'orlo del delirio. La sua fine era già chiara sin dall'inizio e il rifiuto di trattare da parte della maggior parte dei partiti dell'arco costituzionale lo dimostrava.

Un primo esempio di quanto riportato finora si può riscontrare già dal titolo di un articolo apparso sul Corriere della Sera il 30 marzo, «Moro scrive a Cossiga. Quelle parole non sono sue», ventiquattr'ore dopo la diffusione di una lettera di Moro al Ministro degli Interni Cossiga. Nella missiva il prigioniero estendeva a tutto il partito il processo popolare al quale era sottoposto e proponeva un possibile scambio di prigionieri (alcuni militanti delle Brigate Rosse o di altri gruppi armati liberi da delitti di sangue potevano essere messi in libertà e in cambio si sarebbe salvata la vita del politico). La reazione della $\mathrm{DC}$, che si era già posizionata sulla linea della fermezza, mai abbandonata fino alla tragica conclusione della vicenda, fu quella, come si è visto, di sottrarre veridicità alle parole scritte dal collega. La stampa fu la cassa di risonanza di questa posizione intransigente del partito. In quello stesso giorno iniziò la fine del prigioniero. Togliere credibilità a quanto scritto dal sequestrato si accompagnava all'impossibilità di negoziare. Da una parte c'era lo Stato, rappresentato dalla formazione di governo, la Democrazia Cristiana, dall'altra un gruppo di sanguinari assassini. Moro aveva perso credibilità ed era così uscito dalle trattative per salvarsi. Le reazioni di altri quotidiani furono chiare. Il Giornale Nuovo si chiedeva se fosse autentica la lettera ${ }^{41}, L^{\prime}$ Unità la definiva «tragica» ${ }^{42}$. Quando il 4 aprile venne diffuso il secondo scritto del presidente, questa volta indirizzato a Benigno Zaccagnini, segretario della DC, il Corriere della Sera parlò di «lettera dall'inferno» e evidenziò la reazione della direzione del partito che definiva quanto scrit-

\footnotetext{
41 F.D., «Ma è autentica?», Il Giornale Nuovo, 30-III-1978.

42 Paolo Gambescia, «Una tragica lettera», L'Unità, 30-3-1978.
} 
to «non moralmente attribuibile a $\mathrm{Moro}^{43} \gg . L^{\prime}$ 'Unità faceva leva sul fatto che dietro alle parole di Moro non ci fosse «un uomo libero», ma «solo un essere umano in balia dei suoi carnefici» ${ }^{44}$. Il Giornale Nuovo liquidava con troppa superficialità l'intervento del sequestrato; il commento era lapidario: «non abbiamo alcun commento da fare a questa seconda lettera di Moro per il semplice fatto che non è di Moro ${ }^{45}$. Drastica la reazione de La Repubblica, che dichiarava, senza mezzi termini, la morte di un leader:

Venti giorni di «carcere del popolo», venti giorni di totale isolamento dall'esterno, d'interrogatori incalzanti, di continua e brutale pressione psicologica. Questa è la prova terribile cui è stato ed è ancora sottoposto Aldo Moro, una prova che avrebbe fiaccato la psiche, l'autocontrollo, l'equilibrio nervoso di qualsiasi uomo.

E infatti i risultati sono venuti puntuali e gravi. Nella cupa atmosfera del «carcere del popolo», lo statista, il leader politico, non esistono quasi più: è restato un uomo angosciato, sconvolto ${ }^{46}$.

La credibilità di Moro come persona carismatica era svanita nel nulla in meno di venti giorni dal suo sequestro. Uno dei politici più influenti del paese era definitivamente abbandonato a se stesso.

Con il passare dei giorni la stampa cominciò a parlare della possibilità che il sequestrato fosse sedato o drogato. Da parte sua lo statista intensificava la sua personale corrispondenza con gli uomini del suo partito, verso i quali iniziava a provare disagio e rancore. Inquietante è il fatto che la cronaca di quei giorni fosse quasi interamente dedicata a smontare l'immagine e l'integrità di Aldo Moro e non vi fossero novità di altro tipo. I quotidiani iniziarono a riempirsi di articoli di «non informazione». Si ripetevano fino alla noia concetti già espressi, si cercavano interviste sempre più scontate e banali, si rispecchiava fedelmente quanto ottenuto fino a quel momento dalla polizia: il nulla.

Un colpo di scena arrivò il 18 aprile, quando nel comunicato numero sette delle Brigate Rosse si leggeva che Aldo Moro era stato giustiziato e il suo cadavere si trovava nei fondali del Lago della Duchessa, in provincia di Rieti. In realtà non furono in molti a credere che l'esecuzione fosse realmente avvenuta. Il primo indizio che faceva pensare al contrario era che in quel periodo dell'anno il lago era completamente ghiacciato. Le immagini, alquanto patetiche, dei sommozzatori dell'esercito intenti a traforare il ghiaccio e a calarsi a temperature glaciali nella ricerca di un corpo che era impossibile che fosse laggiù sono ancora negli occhi di molti testimoni di allora. Un altro elemento che faceva crollare la versione brigatista era il tono scherzoso della rivendicazione. Nel messaggio si parlava di «esecuzione attraverso suicidio». Le Brigate Rosse, molto scrupolose nella redazione dei loro scritti, non

43 «Una lettera dall'inferno», Corriere della Sera, 5-IV-1978.

44 «Unità nella fermezza», L’Unità, 5-IV-1978.

45 Indro Montanelli, «Il ricatto delle BR», Il Giornale Nuovo, 5-IV-1978.

46 Sandro Viola, «In 20 giorni hanno ucciso un leader», La Repubblica, 5-IV-1978. 
avrebbero mai utilizzato un tono irrisorio nel diffondere la notizia più tragica. Si scoprì in pochi giorni che si trattava di una squallida messa in scena. Il 19 aprile i dubbi sulla veridicità dell'accaduto venivano riportati da più testate giornalistiche. La Repubblica in prima pagina domandava: «Moro Assasinato?», L'Unità utilizzava un eloquente condizionale: «Moro sarebbe stato assasinato», Il Giornale Nuovo manteneva la speranza che fosse «uno scherzo atroce» ${ }^{47}$.

Solo il 20 aprile le Brigate Rosse divulgarono un comunicato in cui si diceva che Moro era ancora vivo e in cui si accusava la messa in scena che era stata organizzata. Tempo dopo si scoprì che gli autori del falso comunicato furono degli agenti segreti italiani che volevano provare l'impatto della tragica notizia che, per altro, da lì a poco si presenterà con tutta la sua veridicità. I brigatisti iniziarono a proporre uno scambio di prigionieri, ma le reazioni di politici e stampa furono intransigenti. Il Corriere della Sera fu chiaro fin dal suo titolo: «La Repubblica non si baratta». Secondo L'Unità non vi doveva essere «nessun cedimento» da parte delle istituzioni. Per Montanelli le condizioni per salvare Aldo Moro erano così assurde da far credere che i brigatisti volessero far ricadere la colpa della morte dello statista sullo Stato italiano. La Repubblica poneva i cittadini di fronte a un terribile dilemma: «Sacrificare un uomo o perdere lo Stato» ${ }^{48}$.

Gli ultimi giorni di aprile furono lo specchio della tragicità di quel periodo. Da una parte Aldo Moro intensificava il suo carteggio, molto duro e accusatorio in alcune circostanze, con Ministri di ogni corrente politica, collaboratori, amici e addirittura con il Papa. Dall'altra parte vi era un muro difficile da abbattere. In mezzo i terroristi che presagivano che quella situazione di stallo in cui si trovavano non poteva continuare all'infinito. Dal giorno del sequestro la polizia non era riuscita a mettere a segno nessun colpo d'effetto, però era evidente che prima o poi qualcosa sarebbe cambiato ed era un rischio protrarre per molto tempo il rapimento e il «processo popolare».

Da parte loro, i mezzi di comunicazione reiteravano l'impossibilità di dare credibilità a quanto scritto da un uomo in quella situazione. E così, valga un esempio fra tutti, quello de L'Unità secondo cui vi era per lo meno una certezza, che «ciò che esce dalla prigione è ciò che pensano e vogliono i carcerieri» ${ }^{49}$. Dallo scoop rivelatosi falso della morte di Moro si dovettero attendere quasi venti giorni prima di assistere a un altro scossone in una vicenda così succulenta per i mezzi di comunicazione. La mattina del 5 maggio le Brigate Rosse diffusero un comunicato in cui dichiaravano che il tempo era ormai scaduto e che la sentenza di morte sarebbe stata realizzata. L'indomani i giornali dipinsero uno scenario apocalittico. $L a$ Repubblica parlava di un possibile «terrorismo di massa congiunto a una sommossa di piazza» e non rasserenava gli animi analizzando il completo fallimento delle

47 «Moro assassinato?», La Repubblica; «Moro sarebbe stato assasinato», L'Unità; Indro Montanelli, «Da un 18 aprile all'altro», Il Giornale Nuovo, 19-IV-1978.

48 «La Repubblica non si baratta», Corriere della Sera; «Nessun cedimento», L'Unità; Indro Montanelli, «Non è sempre carità», Il Giornale Nuovo; «Sacrificare un uomo o perdere lo Stato», La Repubblica, 21-IV-1978.

49 «Le lettere di Moro», L’Unità, 1-V-1978. 
forze dell'ordine che, dopo cinquanta giorni, non erano riuscite a rintracciare nemmeno un indizio su dove si trovasse il sequestrato ${ }^{50}$. Indro Montanelli, dalle pagine de Il Giornale Nuovo, con forte cinismo invitava i suoi lettori a «considerare il caso come avvenuto e chiuderlo». Secondo il giornalista il «dopo Moro» era già cominciato $^{51}$.

La sentenza venne eseguita davvero: il 9 maggio il corpo senza vita di Aldo Moro fu abbandonato dentro un'auto parcheggiata in via Caetani a Roma a poca distanza dalle sedi della Democrazia Cristiana e del Partito Comunista Italiano, nel cuore istituzionale del Paese. I giornali reagirono condannando quanto accaduto, ma allo stesso tempo facendo in modo di guardare verso un futuro migliore. Secondo il Corriere della Sera Moro era morto affinché vivesse la Repubblica, si trattava di una visione salvatrice, quasi catartica dell'accaduto, come se con la morte del presidente della Democrazia Cristiana lo Stato italiano si fosse liberato di un tremendo peso $^{52}$. Il quotidiano socialista Avanti! si augurava un'unione di tutte le persone democratiche per la difesa della Repubblica ${ }^{53}$. Anche secondo La Repubblica il paese doveva reagire compatto alla sfida delle $\mathrm{BR}^{54}$. Così si conclusero i cinquantacinque giorni che andavano dalla strage di via Fani all'omicidio di Aldo Moro. Si era trattato, da un punto di vista giornalistico, di un lasso di tempo caratterizzato da una reiterazione informativa a volte pedante e monotona, da un'intransigente chiusura rispetto alla posizione del presidente della Democrazia Cristiana con la conseguenza dell'abbandono dello statista al suo tragico destino, da una esaltazione costante della potenza strategico-militare delle Brigate Rosse. Ciò di cui non vi era più alcun dubbio, dopo otto anni dalla loro nascita, era la collocazione ideologica delle Brigate Rosse. Se sul loro effettivo valore e pericolo già si era fatta luce in precedenza, a volte esaltandone oltremisura l'efficacia, con quanto accaduto ad Aldo Moro anche i più scettici accetteranno di posizionare i brigatisti all'interno di quell' «album di famiglia» di cui aveva parlato Rossana Rossanda, leader del gruppo extraparlamentare di estrema sinistra Il Manifesto, nonché giornalista dell'omonima pubblicazione quotidiana. Fu lei che, non senza sollevare intense polemiche, dichiarò, proprio durante i due mesi del sequestro, che:

Chiunque era stato comunista negli anni cinquanta riconosce di colpo il nuovo linguaggio delle BR. Sembra di sfogliare l'album di famiglia. Ci sono tutti gli ingredienti che ci vennero propinati nei corsi Stalin e Zdanov di felice memoria [...] Vecchio o giovane che sia il tizio che maneggia 1 ' $\mathrm{Ibm}^{55}$, il suo schema è veterocomunista puro $^{56}$.

50 «Democrazia contro terrorismo», La Repubblica, 5-V-1978.

51 Indro Montanelli, «I registi del brivido», Il Giornale Nuovo, 7-V-1978.

52 Franco di Bella, «È morto perché questa Repubblica viva», Corriere della Sera, 10-V-1978.

53 «Uniti tutti i democratici. Difendiamo la Repubblica», Avanti!, 10-V-1978.

54 «L'assassinio di Moro», La Repubblica, 10-V-1978.

55 Con una Ibm le Brigate Rosse scrissero i comunicati di rivendicazione durante i cinquantacinque giorni del sequestro di Aldo Moro.

56 Rossana Rossanda, «Il discorso sulla DC», Il Manifesto, 28-III-1978. 
Queste parole, così reali e al contempo così difficili da digerire, crearono un certo imbarazzo tra gli ambienti comunisti di quell'epoca. Tuttavia, una volta assunta la consapevolezza che i brigatisti andavano cercati in quell'ambito ideologico e non tra il neofascismo stragista, la stampa iniziò ad essere più obiettiva e precisa nelle sue informazioni. La campagna caotica di disinformazione o di informazione lacunosa sembrava ormai terminata.

\section{QUANDO SI PENSÒ AL BLACK OUT DELL'INFORMAZIONE}

Come si è già anticipato in queste pagine la stampa italiana, durante gli anni settanta, arrivò a proporre, in pochi ed eccezionali casi, la sospensione cautelare delle notizie inerenti al terrorismo brigatista per far sì che i terroristi non manipolassero le informazioni a loro piacimento.

Un mese prima del sequestro di Aldo Moro, il sociologo canadese Marshall McLuhan, interpellato dal giornale Il Tempo, aveva lanciato la provocazione del blackout del sistema informativo, dicendo che senza comunicazione non sarebbe esistito il terrorismo e che quindi la prima cosa da fare sarebbe stato staccare la spina, spegnere la luce ${ }^{57}$.

Il concetto di interruzione volontaria dell'informazione venne ripreso nei primi giorni di prigionia del politico per far sì che i terroristi non manipolassero la stampa italiana. Si aprì un intenso dibattito sull'opportunità o meno di procedere al blackout. Il premio nobel Eugenio Montale, in un'intervista al Corriere della Sera, diceva che i giornalisti dovevano seguire un codice deontologico e non pubblicare tutto quanto arrivava dalla «prigione del popolo» ${ }^{58}$. L'intellettuale distingueva il diritto all'informazione dal fatto di essere utilizzati come cassa di risonanza da un gruppo di criminali. Secondo Lamberto Pignotti de L'Unità bisognava continuare a pubblicare i comunicati brigatisti «ma senza eccedere nel gigantismo e indulgere all'affetto sensazionale della notizia ${ }^{59}$. Giampaolo Pansa da parte sua chiedeva su $L a$ Repubblica, sotto l'emblematico titolo «Silenzio-stampa sul processo a Moro?», responsabilità a tutti i suoi colleghi, senza la necessità di arrivare a un blackout. La posizione de La Repubblica di continuare ad informare, ma di prestare attenzione a non fare il gioco dei terroristi, era quella più diffusa tra la carta stampata. Staccare la spina era impensabile, di fatto i terroristi avevano confezionato una delle notizie più mediatiche della storia della Repubblica italiana. Tuttavia, dopo un inizio trionfale di copertura informativa, molti quotidiani diedero uno spazio eccessivo alla linea della fermezza e accantonarono il povero Aldo Moro che già era stato allontanato dai colleghi di partito.

$\mathrm{Fu}$ invece in concomitanza con un'altra azione brigatista che i giornali riconsiderarono la possibilità di smettere di dare informazioni o per lo meno di ridurne la

57 Intervista di Gino Fantauzzi al sociologo McLuhan, Il Tempo, 19-II-1978.

58 «Un caso di coscienza», Corriere della Sera, 21-III-1978.

59 Lamberto Pignotti, «Il terrorista in prima pagina», L'Unità, 25-III-1978. 
portata. Il 12 dicembre 1980 un commando delle BR sequestrò il giudice Giovanni D'Urso, direttore dell'ufficio III della Direzione Generale degli Istituti di Prevenzione e Pena del Ministero della Giustizia. Per la prima volta nella loro storia le Brigate Rosse cercarono, in parte riuscendovi, di utilizzare in maniera diretta i mezzi di comunicazioni con fini propagandistici. Le BR chiesero al Governo la chiusura del carcere speciale dell'Asinara in cambio della vita del prigioniero. Prima della fine dell'anno la prigione venne chiusa. L'esecutivo, per evitare inutili polemiche, rese noto che la decisione era già stata presa prima del rapimento del giudice e che l'azione violenta dei terroristi non aveva fatto altro che accellerare un'iniziativa già in programma. La spiegazione non convinse del tutto, però in una situazione d'emergenza il buon senso fece sì che si evitassero controversie.

Una volta raggiunto l'obiettivo, i brigatisti non mantennero la parola data e chiesero che i mezzi di comunicazione divulgassero alcuni comunicati scritti da terroristi incarcerati nei penitenziari di Trani e Palmi. Il 4 gennaio le Brigate Rosse condannarono a morte il loro prigioniero. Se si voleva salvare D'Urso bisognava muoversi al più presto. Fu in quel momento che il giornalismo italiano si vide immerso in un grosso dilemma: dare spazio a degli assassini per salvare la vita di un uomo oppure rimanere saldi sulla posizione di fermezza e probabilmente essere in parte responsabili di un omicidio. I mezzi di comunicazione si divisero. Già dal 6 gennaio alcuni quotidiani iniziarono a parlare di blackout. Una delle prime testate che si posizionò in questo senso fu il conservatore Il Tempo che proponeva a grandi caratteri di «Staccare la spina», il direttore de La Repubblica Eugenio Scalfari difese invece il diritto d'informazione, ma allo stesso tempo si rifiuatava di pubblicare $\mathrm{i}$ proclami dei brigatisti, il Corriere della Sera scelse il silenzio. Queste le parole della direzione del giornale:

La Direzione del Corriere della Sera, d'intesa con la Direzione generale del Gruppo editoriale e informato il Comitato di redazione, ha deciso da oggi il completo silenzio stampa sulle richieste dei terroristi rapitori del giudice D’Urso [...] Siamo convinti che il silenzio stampa è l'unica strada per tentare di sottrarre il giudice sequestrato alla tortura di un baratto che non avrebbe mai fine [...] sappia il lettore che questa decisione non lo priverà di alcuna vera notizia: faremo da oggi un giornale, se possibile, ancora più informato eliminando nella cronaca del terrorismo solo quella parte di puro ricatto che tende ad avvelenare e stravolgere la verità trasformando i giornali in strumento d'eversione ${ }^{60}$.

Come possiamo vedere, la reazione della stampa fu diametralmente opposta rispetto a quanto accaduto con Aldo Moro. Tuttavia una posizione di intransigenza totale non era possibile e di fatto ci furono ben presto dei cedimenti. Da parte sua anche Il Giornale Nuovo decise di staccare la spina e avvisò i lettori dicendo «mai più messaggi delle Brigate Rosse» anche se quella decisione, secondo lo stesso direttore del quotidiano, significava «quasi certamente la morte del giudice D'Ur-

60 «Ai lettori: il Corriere ha deciso il silenzio stampa sulle richieste BR», Corriere della Sera, 6-I-1981. 
so» ${ }^{61}$. L'Unità manteneva una posizione di ambiguità sul blackout, dichiarando che avrebbe deciso «in ogni contingenza, se e come utilizzare la conoscenza dei fatti ${ }^{62}$.

Già il giorno 7 il giornalista de Il Manifesto Mauro Paissan aveva dichiarato l'impossibilità di una simile proposta di interruzione dell'informazione. Anche l' $A$ vanti! era scettico sull'efficacia di staccare la spina. Il direttore del quotidiano socialista riteneva che l'unico silenzio utile fosse quello che bisognava applicare alle polemiche e divisioni tra i partiti politici e il mondo dell'informazione ${ }^{63}$. Con la divisione della stampa italiana e con una serie di polemiche ad essa vincolate, si corse il rischio di dimenticare che nel frattempo un uomo stava rischiando la propria vita e che su di lui pesava una condanna a morte. L'Avanti! decise di pubblicare una parte dei comunicati dei prigionieri brigatisti, ma non fu sufficiente e la reazione dei criminali fu immediata, nel comunicato del 10 di gennaio si dava ai mezzi di comunicazione del paese un ultimatum di 48 ore per pubblicare gli scritti, altrimenti la sentenza sarebbe stata eseguita. Le reazioni alle minacce brigatiste furono molto diverse. Il Giornale Nuovo manteneva la sua linea dura considerando D'Urso già uomo morto e quindi non piegandosi alle richieste dei terroristi ${ }^{64}$. Scalfari in La Repubblica rifiutava «il ricatto in nome di altre vite» ${ }^{65}$. Il Corriere della Sera dichiarava che «non si negozia con nemici feroci e spietati» ${ }^{66}$. L'Unità non perse tempo e accusò i socialisti di debolezza dal momento che questi avevano acconsentito a pubblicare una parte dei comunicati dei terroristi ${ }^{67}$. Nella stessa posizione dell'Avanti! (organo di stampa del Partito Socialista appunto) vi erano anche Lotta Continua, Il Manifesto, Il Secolo XIX, Il Lavoro. Ma furono i radicali, anch'essi propensi ad assecondare la richiesta dei terroristi piuttosto che avere sulla coscienza una vita umana, che, il 13 gennaio, durante il programma televisivo Tribuna Politica, cedettero una parte del loro tempo alla figlia del giudice D'Urso affinché leggesse una parte di un comunicato in cui, tra le altre cose, il padre veniva definito boia ${ }^{68}$.

L'indomani, tra le numerose polemiche che si erano sollevate dopo che un'adolescente si era umiliata davanti alle telecamere per poter salvare la vita al padre, $i$ quotidiani allineati a favore della diffusione di quanto richiesto dalle Brigate Rosse decisero di pubblicare i comunicati. I terroristi, sentendosi vincitori di questa lunga battaglia mediatica, rispettarono i patti e liberarono il prigioniero. Per la prima volta, e anche ultima, un gruppo di criminali era riuscito a dirigere l'informazione di un

61 «Uomini e topi», Il Giornale, 6-I-1981.

62 Comunicato in prima pagina in L'Unità, 6-I-1981.

63 Ugo Intini, «Il silenzio più utile è su quanto esaspera divisioni e tensioni», Avanti!, 6-1-1981.

64 Indro Montanelli, «Con angoscia ma No», Il Giornale, 11-I-1981.

65 Eugenio Scalfari, «No al ricatto in nome di altre vite», La Repubblica, 12-I-1981.

${ }^{66}$ Leo Valliani, «I ricattatori di fronte alle loro resonsabilità», Corriere della Sera, 11-I-1981.

67 «Per salvare la vita di tutti», L'Unità, 11-I-1981.

68 In realtà, secondo le testimonianze del radicale Franco Spadaccia al giornalista Sergio Zavoli, il Partito Radicale aveva ceduto una parte del suo tempo affinché la famiglia D'Urso facesse un appello di clemenza ai rapitori, all'improvviso però, la figlia estrasse dalla tasca un foglio e iniziò a leggere il comunicato dei terroristi. 
paese democratico e libero come l'Italia. Mai più nessuno, fortunatamente, riuscì a emulare quanto ottenuto dalle $\mathrm{BR}$ in quella circostanza, però le polemiche che si crearono all'interno della stampa italiana proseguirono per molto tempo.

\section{RIFERIMENTI BIBLIOGRAFICI}

BENITO, Ángel (1986): «Terrorismo y medios de comunicación», en Escritos sobre la tolerancia, Madrid, Editorial Pablo Iglesias, pp. 113-138.

Brambilla, Michele (1991): L'eskimo in redazione, Milano, Edizioni Ares.

CAtAnzaro, Raimondo (1990): Ideologie, movimenti, terrorismi, Bologna, Il Mulino.

Clementi, Marco (2007): Storia delle Brigate Rosse, Roma, Obradek Edizioni.

Eco, Umberto (1977): Dalla periferia dell'Impero, Milano, Bompiani. [Trad. sp. di Eduardo Oviedo, La estrategia de la ilusión, Barcelona, Lumen, 1986].

FrANCESCHINI, Alberto (1988): Mara, Renato e io, Milano, Mondadori Editore.

GaLli, Giorgio (2004): Piombo rosso, Milano, Baldini Castoldi Dalai.

LAQUEUR, Walter (1978): Storia del terrorismo, Milano, Rizzoli.

SÁNCHEZ RODRíGUEZ, Gabriel (2005): «Informar sobre terrorismo: una misión difícil pero necesaria», Comunicación y hombre I, pp. 71-90.

SATTA, Vladimiro (2003): Odissea nel caso Moro, Roma, EDUP.

Soccorso Rosso (1976): Brigate Rosse. Che cosa hanno fatto, che cosa hanno detto, che cosa se ne è detto, Milano, Feltrinelli.

TESSANDORI, Vincenzo (2004): Imputazione: banda armata, Milano, Baldini Castoldi Dalai. VERES, Luis (2002): «El signo perverso: sobre lenguaje, terrorismo y práctica periodística», Revista Latina de Comunicación Social VII, pp. 1-5.

Veres, Luis (2005): «La simbiosis del terrorismo con los medios de comunicación», en Información para la paz: autocrítica de los medio y responsabilidad del público, Valencia, Fundación COSO, pp. 583-601.

Zavoli, Sergio (1992): La notte della Repubblica, Milano, Mondadori Editore. 